\title{
Suppression of left-handed properties in disordered metamaterials
}

\author{
Alexander A. Zharov ${ }^{1,2}$, Ilya V. Shadrivov ${ }^{1}$, and Yuri S. Kivshar ${ }^{1}$ \\ ${ }^{1}$ Nonlinear Physics Centre, Research School of Physical Sciences and Engineering, \\ Australian National University, Canberra ACT 0200, Australia \\ 2 Institute for Physics of Microstructures, Russian Academy of Sciences, Nizhny Novgorod 603950, Russia
}

\begin{abstract}
We study the effect of disorder on the effective magnetic response of composite left-handed metamaterials and their specific properties such as negative refraction. We show that relatively weak disorder in the split-ring resonators can reduce and even completely eliminate the frequency domain where the composite material demonstrates the left-handed properties. We introduce the concept of the order parameter to describe novel physics of this effect.
\end{abstract}

Recently fabricated composite structures [1, 2, 3] open an unique possibility to study experimentally the properties of the so-called left-handed metamaterials [4]. Specifically, the composite materials created by arrays of wires and split-ring resonators (SRRs) are known to possess a negative real part of the magnetic permeability and negative dielectric permittivity in the microwave range [1, 2, 3], and they demonstrate many unusual properties predicted theoretically long time ago [4], including negative refraction, inverse light pressure, reverse Doppler and Vavilov-Cherenkov effects, etc. Moreover, many ideas to create such left-handed metamaterials for optical frequencies are currently under study (see, e.g., Ref. [5]).

The metamaterials created by arrays of metallic wires and microwave resonators are designed to operate for the wavelengths much larger then the period of the structure, and their simultaneously negative dielectric permittivity and magnetic permeability occur in some finite frequency range. Within the effective medium approximation, dielectric permittivity becomes negative in a relatively large frequency domain due to a linear response of wires, whereas magnetic permeability of the structure can become negative in a relatively narrow frequency domain. It is this specific frequency domain where the induced magnetic momentum of each resonator is directed opposite to the external magnetic field being strong enough to produce collectively negative values of the effective magnetic permeability.

In this Letter, we study the effect of weak disorder in the structure parameters on the magnetic response of composite and the existence of the frequency domain where the composite material exhibits the specific lefthanded properties such as negative refraction. We show that even relatively small variation of the SRR parameters can result in the dramatic decrease of the size of the left-handed frequency domain and, above a certain threshold value, disorder can suppress and even eliminate completely the left-handed properties of the metamaterial.

We study a composite structure made of metallic wires and single-ring microwave resonators, as shown schematically in Fig. I(a). This model is qualitatively similar to the model of double-ring resonators usually studied in the theory of left-handed composite media (see, e.g., Ref. [] and reference therein). Within the effective medium approximation, the dielectric permittivity of this structure can be calculated [7, [8] and presented in the form

$$
\epsilon_{\mathrm{eff}}(\omega) \approx 1-\frac{\omega_{p}^{2}}{\omega\left(\omega-i \gamma_{e}\right)},
$$

where $\omega_{p}=2 \pi c / d \ln \left(d / r_{w}\right), d$ is the size of the unit cell, $r_{w}$ is the radius of a metallic grid, $\gamma_{e}=c^{2} / 2 \sigma S \ln \left(d / r_{w}\right)$, $\sigma$ is conductivity of a wire, $S$ is the effective wire crosssection, $\omega$ is the angular frequency, and $c$ is the free-space speed of light. Usually, the effective plasma frequency, $f_{p}=\omega_{p} / 2 \pi$, is between $10 \mathrm{GHz}$ and $15 \mathrm{GHz}$.

The most intriguing properties of the left-handed composite metamaterials are due to a SRR response which determines effective magnetic permeability. Magnetization of the metamaterial with three SRRs per unit cell, each of them having the axis in the perpendicular direction with respect to others as shown in Fig. M(a), can be presented in the form (see, e.g., Refs. 9, 10]), $\mathbf{M}=\chi(\omega) \mathbf{H}^{\prime}$, where

$$
\begin{gathered}
\chi(\omega)=\frac{\eta \omega^{2}}{\omega_{0}^{2}-\omega^{2}+i \gamma_{m} \omega}, \\
\eta=\frac{\pi}{8}\left(\frac{a}{d}\right)^{3}[\ln (8 a / r)-7 / 4]^{-1 / 2} .
\end{gathered}
$$

Here, $\quad \mathbf{H}^{\prime}$ is acting (microscopic) magnetic field, $\quad \omega_{0}=\left[d_{g} c^{2} / \pi \epsilon r^{2} a(\ln (8 a / r)-7 / 4)\right]^{1 / 2}$ is the SRR eigenfrequency, $a$ is a SRR radius, $\gamma_{m}=c^{2} / 2 \sigma_{r} S_{r}[\ln (8 a / r)-7 / 4]$ is damping coefficient, $d_{g}$ is the size of the SRR slot, $\epsilon$ is permittivity of the dielectric infilling the SRR slot in the structure.

Results (2) and (3) are obtained under the major assumption that all resonators in the structure are identical. Now we consider the case when the size of the slot $d_{g}$ is random and it is characterized by a statistical distribution function. Our study can be motivated by a number of the recent efforts to create the metamaterials with the left-handed properties for shorter wavelengths and, for the smaller SRR size in the structure, the key parameters would be difficult to control in fabrication. In our structure, the parameter $\eta$ does not depend on $d_{g}$ [see Eq. (32] ], and only the SRR eigenfrequency $\omega_{0}$ is 
affected by fluctuations of $d_{g}$. Then, the equation for the magnetic susceptibility (2) can be generalized to describe the case of randomly varying eigenfrequency,

$$
\chi(\omega)=\eta \omega^{2} \int_{0}^{\infty} \frac{F(X) d X}{X^{2}-\omega^{2}+i \gamma_{m} \omega},
$$

where $F(X)$ is the normalized distribution function of the SRR eigenfrequencies, i.e. $\int_{0}^{\infty} F(X) d X=1$. In the standard case when all SRRs are identical, the distribution function can be represented as $F(X)=\delta\left(X-\omega_{0}\right)$.

To describe the coupling between the acting magnetic field $\mathbf{H}^{\prime}$ and the macroscopic magnetic field $\mathbf{H}$, we use the Lorentz-Lorenz formula 11], $\mathbf{H}^{\prime}=\mathbf{H}+(4 \pi / 3) \mathbf{M}$, and present the effective magnetic permeability in terms of local magnetic susceptibility (4) as follows

$$
\mu_{\mathrm{eff}}(\omega)=\frac{1+(8 \pi / 3) \chi(\omega)}{1-(4 \pi / 3) \chi(\omega)}
$$

For definiteness, we consider the Lorenz-type distribution of the SRR eigenfrequencies in the form

$$
F(X)=\frac{(\Gamma / \pi)}{\left(X-\omega_{0}\right)^{2}+\Gamma^{2}},
$$

with a narrow width for $\Gamma \ll \omega_{0}$ such that the eigenfrequencies of all resonators are close to some mean value $\omega_{0}$. As a result, non-vanishing contributions to the integral are given by the values of $X$ in the vicinity of $X=\omega_{0}$, and we introduce a new variable $\Delta, X=\omega_{0}+\Delta$, where $|\Delta| \ll \omega_{0}$. We are interested in the behavior of the magnetic susceptibility in the vicinity of $\omega=\omega_{0}$, and we introduce $\omega=\omega_{0}+\Omega$, where $|\Omega| \ll \omega_{0}$. In this approximation, Eq. (4) can be rewritten as

$$
\chi(\omega) \approx \frac{\eta}{2 \pi} \omega_{0} \Gamma \int_{-\infty}^{\infty} \frac{d \Delta}{\left(\Delta^{2}+\Gamma^{2}\right)\left(\Delta-\Omega+i \gamma_{m} / 2\right)} .
$$

Using the contour integration, the integral in Eq. (17) can be calculated explicitly, and the expression for the magnetic susceptibility can be obtained in the following form:

$$
\chi(\Omega)=\frac{\omega_{0} \eta}{2} \frac{1}{(-\Omega+i \bar{\Gamma})},
$$

where $\bar{\Gamma}=\Gamma+\gamma_{m} / 2$. The result (8) shows that the random variation of the SRR eigenfrequencies is equivalent to some additional losses in the structure, and even for an ideal case of lossless resonators, i.e., when $\gamma_{m}=0$, the composite structure with random variation of the SRR frequencies possesses effective losses. From the physical point of view, such effective losses resemble the collisionless Landau damping in plasmas [12] caused by the presence of resonant particles and, simultaneously, it follows from the Kramers-Kronig relations.

From Eqs. (51) and (8), we find the frequency domain where the real part of magnetic permeability becomes negative, $\Omega_{1}<\Omega<\Omega_{2}$, where

$$
\Omega_{1,2}=\frac{1}{3} \Gamma_{c} \mp \sqrt{\Gamma_{c}^{2}-\bar{\Gamma}^{2}},
$$

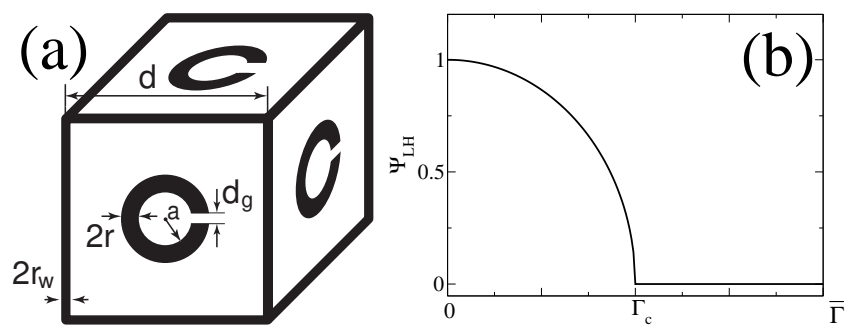

FIG. 1: (a) Schematic of the metamaterial structure. (b) Dependence of the real part of the order parameter $\Psi_{\mathrm{LH}}$ on the effective combined losses $\bar{\Gamma}$ in the composite structure.

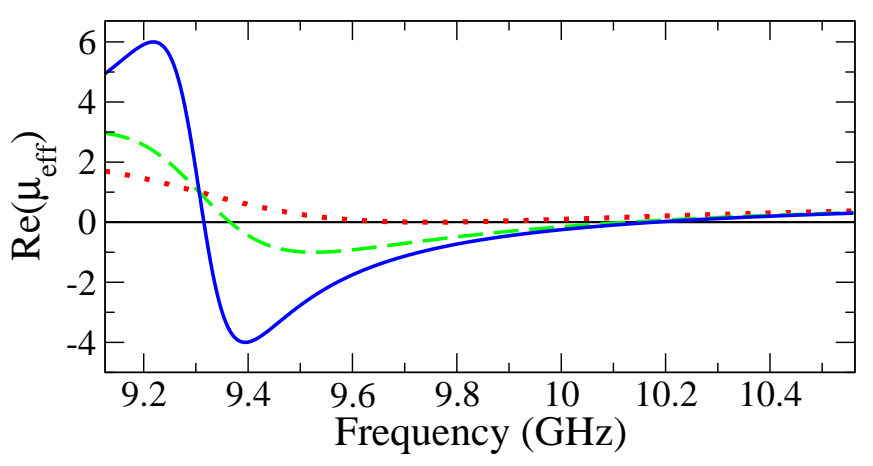

FIG. 2: Real part of the effective magnetic permeability as a function of the wave frequency for $\bar{\Gamma} / \Gamma_{c}=0.2$ (solid), $\bar{\Gamma} / \Gamma_{c}=$ 0.5 (dashed), and $\bar{\Gamma} / \Gamma_{c}=1$ (dotted).

and $\Gamma_{c}=\pi \eta \omega_{0}$. Thus, the width of the frequency domain of the negative effective magnetic permeability is

$$
\Delta \Omega_{\mathrm{LH}}=2 \sqrt{\Gamma_{c}^{2}-\bar{\Gamma}^{2}} .
$$

The value $\Gamma_{c}$ has the meaning of the critical losses in the composite structure above which the domain with negative values of the magnetic permeability disappears, and the relative critical parameter $\Gamma_{c} / \omega_{0}$ depends only on the structure of the composite. Taking the characteristic values $d=0.5 \mathrm{~cm}, a=0.2 \mathrm{~cm}$, and $r=0.05 \mathrm{~cm}$, we obtain $\Gamma_{c} / \omega_{0}=0.046$. This result indicates that even for the case of lossless resonators, i.e. $\gamma_{m}=0$, relative fluctuations of the SRR eigenfrequencies cannot exceed $4.6 \%$. Accordingly, if the slot size is $d_{g}=0.01 \mathrm{~cm}$, (then $\omega_{0} / 2 \pi=9.6 \mathrm{GHz}$ ) the critical variation can be estimated as $\Delta d_{g} \sim 5 \mu \mathrm{m}$. Existence of such a critical distribution width places strict requirements on the manufacturing of such materials. Although for microwaves such requirements can be easily met, they can result in substantial experimental difficulties for shorter wavelengths. In particular, the recent proposal to fabricate left-handed metamaterials in optics using nanowires and $\pi$-shaped particles [5], would require an accuracy better than $5 \mathrm{~nm}$, that can be challenging for the existing technology.

We note that premeditative introduction of disorder does not allow to increase the size of the left-handed frequency domain for any type of the distribution function. 
Such a conclusion has a simple physical explanation. Indeed, disorder in the eigenfrequencies for any given SRR density results in a decrease of the effective number of resonators, which contribute into the negative magnetization. In the recent experiment [13], combining s-shaped resonance particles, the authors fabricated the metamaterial with two domains of the negative refraction. Using the results obtained above, we may come to the conclusion that the total size of the frequency domain with the negative magnetic permeability in the structure fabricated in Ref. [13] is less than it would be for the metamaterial where all resonators are identical. Moreover, using the resonators with several different values of the eigenfrequency can eliminate completely the left-handed properties.

Reduction and complete suppression of the frequency domain with negative magnetic permeability with a growth of the value of $\bar{\Gamma}$ can also be explained in a different way. Indeed, the left- and right-handed properties of the metamaterial can be treated as two different "phase states" of the structure. Thus, a transition from one state to the other can be interpreted as the phase transition of the second kind. The parameter $\bar{\Gamma}$ describes the effect of disorder in the structure, and it can be treated as "effective temperature". Such comparison is adequate since the effective temperature is determined as the width of statistical frequency fluctuations including both homogeneous and inhomogeneous line broadening. To describe different phase states of the structure, we introduce the effective order parameter, as is usually done in the theory of phase transitions. In the absence of homogeneous $\left(\gamma_{m}=0\right)$ and inhomogeneous $(\Gamma=0)$ SRR line broadening (i.e. in the absence of disorder), the metamaterial has the maximum width of the left-handed domain, which decreases and then disappears at the critical "effective temperature" $\Gamma_{c}$ termed as the temperature of the phase transition. We use the ratio of the left-handed frequency range to the maximum left-handed frequency domain as the order parameter,

$$
\Psi_{\mathrm{LH}}=\Delta \Omega_{\mathrm{LH}} / \Delta \Omega_{\mathrm{LH}}^{(\max )}=\operatorname{Re}\left[1-\left(\bar{\Gamma} / \Gamma_{c}\right)^{2}\right]^{1 / 2} .
$$

so that the metamaterial possesses the left-handed properties when $\Psi_{\mathrm{LH}} \neq 0$, i.e., below the effective critical temperature $\Gamma_{c}$. Dependence of the order parameter $\Psi_{\mathrm{LH}}$ on
$\bar{\Gamma}$ is shown in Fig. 1(b). The real part of the magnetic permeability as a function of the wave frequency is shown in Fig. 2 for different values of the ratio $\bar{\Gamma} / \Gamma_{c}$. For large values of $\bar{\Gamma} / \Gamma_{c}$, the frequency domain where the material possesses negative magnetic permeability is eliminated due to disorder.

The result (11) suggests two ways for increasing the width of the effective frequency domain where the composite material possesses the left-handed properties, namely, (i) decreasing the effective temperature $\bar{\Gamma}$, e.g. by improving the manufacturing technology for shorter wavelengths, and (ii) increasing the effective critical temperature $\Gamma_{c}$, e.g. by a design of the resonators.

Though the concept of the order parameter $\Psi_{\mathrm{LH}}$ does not seem to be important in the context of the problem under study, it is expected to be useful in other (e.g. nonlinear) problems, when it becomes dependent on the field intensity, time, and coordinate. In this case, the order parameter will determine the material properties in different domains, describing the dynamics of the phase transitions between the states with left- and right-handed properties [8]. The study of the nonlinear regime will be presented elsewhere.

In conclusion, we have analyzed the effect of disorder in the composite structures which exhibit left-handed properties. In particular, we have studied how random variation of the SRR eigenfrequencies in the structure can change the existence of the frequency domain where the effective dielectric permittivity and magnetic permeability are both negative. We have demonstrated that even relatively weak disorder in the SRR parameters can result in a dramatic reduction of the size of the lefthanded frequency domain. More importantly, above a certain threshold value of this disorder, the left-handed frequency domain can disappear completely. We believe our result provide a useful guide for a design of novel types of metamaterials operating for shorter wavelengths where the effect of disorder is expected to be crucially important.

A.A.Z. acknowledges a partial support from the Russian Foundation for Basic Research (grant 05-02-16357) and thanks Nonlinear Physics Centre at the Australian National University for hospitality and fellowship.
[1] D. R. Smith, W. J. Padilla, D. C. Vier, S. C. Nemat Nasser, and S. Schultz, Phys. Rev. Lett. 84, 4184 (2000).

[2] M. Bayindir, K. Aydin, E. Ozbay, P. Markos, and C. M. Soukoulis, Appl. Phys. Lett. 81, 120 (2002).

[3] C. G. Parazzoli, R. B. Greegor, K. Li, B. E. C. Koltenbah, and M. Tanielian, Phys. Rev. Lett. 90, 107401 (2003).

[4] V. G. Veselago, Usp. Fiz. Nauk 92, 517 (1967) [Sov. Phys. Usp. 10, 509 (1968)].

[5] V. A. Podolskiy, A. K. Sarychev, and V. M. Shalaev,
Opt. Express 11, 735 (2003).

[6] P. Markos and C. M. Soukoulis, Phys. Rev. E 65, 036622 (2002).

[7] J. B. Pendry, A. J. Holden, D. J. Robbins, and W. J. Stewart, IEEE Trans. Microw. Theory Tech. 47, 2075 (1999).

[8] A. A. Zharov, I. V. Shadrivov, and Yu. S. Kivshar, Phys. Rev. Lett. 91, 037401 (2003).

[9] J. Schwinger, Classical Electrodynamics (Perseus Books, Reading, Mass., 1998). 
[10] I. V. Shadrivov, N. A. Zharova, A. A. Zharov, and Yu. S. Kivshar, Phys. Rev. E 70, 046615 (2004).

[11] M. Born and E. Wolf, Principles of Optics: Electromagnetic Theory of Propagation, Interference and Diffraction of Light, seventh ed. (Cambridge Univ. Press, 2002).

[12] E. M. Lifshitz and L. P. Pitaevskii, Physical Kinetics
(Pergamon Press, Oxford, 1981).

[13] H. Chen, L. Ran, J. Huangfu, X. Zhang, K. Chen, T. M. Grzegorczyk, and J. A. Kong, J. Appl. Phys. 96, 5338 (2004). 